\title{
Enseñanza de psicoterapia en el pregrado de escuelas de medicina del Perú
}

\section{Teaching of psychotherapy in schools of medicine in Peru}

\author{
Rubén Valle $e^{1,2, a}$, Alberto Perales 3 ,b \\ ${ }^{1}$ Centro de Investigación en Epidemiologia Clínica y Medicina Basada en Evidencias, Facultad de Medicina Humana, Universidad de San Martín de Porres. Lima, Perú. \\ ${ }^{2}$ Dirección Ejecutiva de Investigación, Docencia y Atención Especializada en Adultos y Adultos Mayores, Instituto Nacional de Salud Mental "Honorio Delgado - Hideyo \\ Noguchi". Lima, Perú. \\ ${ }^{3}$ Facultad de Medicina, Universidad Nacional Mayor de San Marcos. Lima, Perú. \\ ${ }^{a}$ Médico psiquiatra, maestro en ciencias de investigación epidemiológica. ORCID: https://orcid.org/0000-0003-0811-200X \\ ${ }^{\mathrm{b}}$ Médico psiquiatra, ORCID: https://orcid.org/0000-0002-3940-5182
}

\begin{abstract}
Correspondencia:
Rubén Valle

ruben_vr12@hotmail.com

Recibido: 11 de abril 2020

Aprobado: 15 de mayo 2020

Publicación en línea: 20 de junio 2020

Conflictos de interés: Los autores declaran no tener conflictos de interés.

Fuente de financiamiento:

Autofinanciado

Contribuciones de autoría: $R V$ concibió y diseño el estudio, recolectó los datos, analizó e interpretó los resultados y redactó el artículo. AP revisó el artículo y aprobó la versión final del manuscrito.
\end{abstract}

Citar como: Valle $R$, Perales $A$. Enseñanza de psicoterapia en el pregrado de escuelas de medicina del Perú. An Fac med. 2020;81(2):2623. DOI: https://doi.org/10.15381/ anales.v81i2.17680
An Fac med. 2020;81(2):262-3. / DOI: https://doi.org/10.15381/anales.v81i2.17680

\section{Sr. Editor,}

La psicoterapia es una herramienta terapéutica basada en la relación empática entre el paciente y su terapeuta, que utiliza técnicas actitudinales y verbales para lograr la recuperación de la salud ${ }^{(1)}$. Los estudios evidencian su eficacia, y guías de práctica clínica la recomiendan en el tratamiento de problemas específicos de salud mental en el primer nivel de atención de salud ${ }^{(2,3)}$. Esto significa que los médicos que trabajan en este nivel debieran conocer las indicaciones y uso de esta valiosa herramienta terapéutica. En el Perú, un gran porcentaje de médicos que trabajan en este primer nivel son médicos recién egresados que realizan el Servicio Rural Urbano Marginal. Estos profesionales atenderán problemas prevalentes de salud mental, tales como depresión, ansiedad y problemas de violencia en su primera experiencia como profesionales de salud ${ }^{(4)}$. Por ello, resulta importante que las facultades de medicina capaciten adecuadamente a los estudiantes de medicina en psicoterapia ${ }^{(1)}$.

Las facultades de medicina de universidades de Estados Unidos y Europa brindan capacitación en psicoterapia a sus estudiantes de pregrado dentro del curso de psiquiatría. El objetivo es que los estudiantes adquieran conocimientos y habilidades en un nivel básico de las corrientes psicoterapéuticas que cuentan con mayor evidencia científica. Con el fin de brindar la mayor cantidad de conocimientos en el menor tiempo, las clases se organizan en reuniones virtuales y presenciales, que incluyen sesiones de videos, juego de roles y grabaciones de tratamiento psicoterapéutico ${ }^{(5)}$. Se ha señalado que la enseñanza de la psicoterapia a los futuros médicos es muy importante en su formación ya que les permitirá establecer relaciones empáticas con sus pacientes, manejar con ecuanimidad las reacciones de transferencia y contratransferencia que inevitablemente se darán en la práctica clínica, y saber cuándo derivar oportunamente a un paciente a este tratamiento ${ }^{(5,6,7)}$.

Con el objetivo de conocer la enseñanza de la psicoterapia en las facultades de medicina del Perú, se evaluaron los syllabus del curso de psiquiatría de diferentes semestres académicos. Los syllabus fueron extraídos de las páginas web de las universidades, o solicitados a profesores de los cursos. Se tuvo acceso a 9 syllabus de psiquiatría de las 45 facultades de medicina del Perú. Los resultados muestran que solo 5 de 9 facultades de medicina impartieron 
Tabla 1. Enseñanza de psicoterapia dentro del curso de psiquiatría de nueve escuelas de medicina del Perú.

\begin{tabular}{|c|c|c|c|c|}
\hline $\begin{array}{l}\text { Universidad } \\
\text { (Gestión) }\end{array}$ & Nombre del curso de psiquiatría (año) & $\begin{array}{l}\text { Número de créditos (horas } \\
\text { académicas) }\end{array}$ & Número de horas de psicoterapia & Porcentaje (\%) \\
\hline Privada & Semiología psiquiátrica y psiquiatría (2019) & $5(80)$ & Lectura, debate y videos (5 horas) & 6,3 \\
\hline Privada & Psiquiatría (2019) & $4(64)$ & Ninguna & 0 \\
\hline Privada & Psiquiatría (2019) & $5(80)$ & Ninguna & 0 \\
\hline Pública & Semiología psiquiátrica y psiquiatría (2018) & $8(128)$ & Teoría: 1,5 Video foro: 3 & 3,5 \\
\hline Privada & Psiquiatría (2018) & $3(48)$ & Teoría: 1,5 & 3,1 \\
\hline Pública & Psiquiatría (2017) & $2(32)$ & Ninguna & 0 \\
\hline Privada & Clínica psiquiátrica (2016) & $6(96)$ & Ninguna & 0 \\
\hline Pública & Psiquiatría (2013) & $5(80)$ & Seminario: 1 & 1,3 \\
\hline Pública & Psiquiatría (2013) & $4(64)$ & Seminario: 2 & 3,1 \\
\hline
\end{tabular}

clases en psicoterapia dentro del curso de psiquiatría, y que el número promedio de horas asignadas a esta materia fue de 1 a 5 horas, lo que representó el 3,1\%$6,5 \%$ del total de horas del curso. Las modalidades de enseñanza incluyeron clases teóricas, seminarios y video foros. Aunque estos resultados no se pueden extrapolar a los cursos de psiquiatría de todas las facultades de medicina del Perú, los hallazgos sugieren que la capacitación en psicoterapia a estudiantes de medicina es escasa y en algunos casos inexistente (Tabla 1).

Estos resultados plantean importantes implicancias sobre la formación de los estudiantes de medicina en el área de la salud mental, particularmente en una época en que la deshumanización de la medicina surge como problema central ${ }^{(8)}$ y se reclama, sobre bases éticas y científicas, un retorno a ubicar a la persona-no sólo a la enfermedad- como su objetivo central ${ }^{(9)}$. Primero, los estudiantes tendrían un aprendizaje insuficiente sobre el tratamiento de la patología mental, orientándolo a un enfoque exclusivamente biológico y psicofarmacológico, desconociendo la existencia de otras estrategias eficaces. Segundo, tal práctica conduciría al estudiante a un concepto reduccionista de la patología mental como de naturaleza exclusivamente bio- lógica obviando la importancia de los factores psicológicos y sociales en la génesis y mantenimiento de la patología mental. Finalmente, la falta de enseñanza de psicoterapia en el pregrado refleja una formación académica incompleta en el campo de la salud mental.

La escasa (o nula) formación en psicoterapia en las facultades de medicina de Perú resulta incongruente con las indicaciones actuales de esta herramienta terapéutica ${ }^{(2)}$. Las facultades de medicina deben fortalecer la enseñanza de psicoterapia en el pregrado, nosotros particularmente sugerimos la enseñanza de psicoterapia de soporte, por ser una corriente que cuentan con alta evidencia científica, su enseñanza no requiere de conocimientos previos en las bases de la psicoterapia, y porque puede ser utilizada por los médicos egresados en el manejo de los síntomas mentales que surgen en el contexto de enfermedades no psiquiátricas ${ }^{(6)}$. La modalidad de enseñanza (presencial o virtual), duración y contenido, son aspectos que cada facultad debe organizar en función a sus recursos (créditos disponibles, profesores capacitados, orientación del curso) con fines que los médicos egresados puedan conocer y manejar casos específicos de patología mental.

\section{REFERENCIAS BIBLIOGRÁFICAS}

1. Perales A. Psicoterapia de Apoyo. En: Manual de Psiquiatria "Humberto Rotondo." Lima: Universidad Nacional Mayor de San Marcos; 1998.

2. Instituto de Evaluación de Tecnologias en Salud e Investigación, Seguro Social de Salud EsSalud. Guia de Práctica Clínica para el tamizaje y el manejo del episodio depresivo leve en el primer nivel de atención: Guia en Versión Corta. Lima: EsSalud; 2019.

3. Parikh SV, Quilty LC, Ravitz P, Rosenbluth M, Pavlova B, Grigoriadis S, et al. Canadian Network for Mood and Anxiety Treatments (CANMAT) 2016 Clinical Guidelines for the Management of Adults with Major Depressive Disorder: Section 2. Psychological Treatments. Can J Psychiatry Rev Can Psychiatr. 2016;61(9):524-39. DOI: https://doi. org/10.1177/0706743716659417

4. Instituto Nacional de Salud Mental "Honorio Delgado- Hideyo Noguchi”. Estudio Epidemiológico de Salud Mental en Lima metropolitana y CallaoReplicación 2012. Lima: Instituto Nacional de Salud Mental "Honorio Delgado- Hideyo Noguchi”; 2013.

5. Aboul-Fotouh F, Asghar-Ali AA. Therapy 101: a psychotherapy curriculum for medical students. Acad Psychiatry J Am Assoc Dir Psychiatr Resid Train Assoc Acad Psychiatry. 2010;34(4):248-52. DOI: 10.1176/appi.ap.34.4.248

6. Perales A. ¿Qué es la psicoterapia de apoyo?. Diagnóstico. 1979;4(3):159-64.

7. Bender EP. Teaching Medical Students Psychodynamic Psychotherapy: An Interactive Method. Acad Psychiatry J Am Assoc Dir Psychiatr Resid Train Assoc Acad Psychiatry. 2016;40(2):396-7. DOI: 10.1007/s40596-015-0481-z

8. Perales A. Deshumanización de la medicina o deshumanización de la sociedad. Lima: Academia Nacional de Medicina; 2018.

9. Perales A. Medicina centrada en la persona: del concepto a la acción. Rev Peru Med Exp Salud Publica. 2016;33(4):605-6. DOI: 10.17843/rpmesp.2016.334.2541. 